\title{
Successful implantation of autologous muscle-derived stem cells in treatment of faecal incontinence due to external sphincter rupture
}

\author{
Romaniszyn Michal • Rozwadowska Natalia • \\ Nowak Marcin • Malcher Agnieszka • \\ Kolanowski Tomasz • Walega Piotr • Richter Piotr • \\ Kurpisz Maciej
}

Accepted: 20 March 2013 / Published online: 3 April 2013

(C) The Author(s) 2013. This article is published with open access at Springerlink.com

\section{Dear Editor:}

The most common pathological mechanism of faecal incontinence is the insufficiency of the external anal sphincter (EAS) caused by neurological or myogenic dysfunction. The myogenic mechanism of EAS insufficiency is usually due to direct mechanical damage during childbirth, trauma or surgery in anorectal region, whereas neurological aetiology involves either spinal or peripheral nerves disruption - in most cases the pudendal nerve. Unfortunately, coincidence of sphincter rupture with damage to pudendal nerves is quite common.

Each skeletal muscle, including EAS, has the ability to regenerate to some degree and repair sustained damage. In response to injury and/or muscle damage, so-called satellite cells are activated and become myoblasts - capable of intense proliferation. Myoblasts then differentiate and fuse together to form new muscle fibres and connect with existing ones, adding new portions of contractile tissue to existing motoric units [1].

Attempts of autotransplantation of myoblasts into damaged skeletal muscle were already made in animal models of muscular dystrophy, post-infarction myocardial dysfunction and urethral sphincter insufficiency [2]. The results showed that the transplanted myoblasts differentiate into muscle fibres, connect with host motoric units, increase the amount

R. Michal $(\triangle) \cdot$ N. Marcin $\cdot$ W. Piotr $\cdot$ R. Piotr

3rd Department of General Surgery, Jagiellonian University

Medical College, Krakow, Poland

e-mail: m.romaniszyn@mp.pl

R. Natalia $\cdot$ M. Agnieszka $\cdot$ K. Tomasz $\cdot$ K. Maciej Department of Reproductive Biology and Stem Cells, Institute of Human Gelnetics, Polish Academy of Sciences, Poznan, Poland of contractile elements in the muscle and improve its contractile activity. In 2001, Menasche et al. first transplanted autologous myoblasts into the post-infarction myocardial scar in human patients with cardiac failure, with significant improvements in contractile function and clinical condition [3]. In Poland, the method of treating post-infarction heart failure was performed for the first time a year later, with similar results [4].

Based on those encouraging results, a pioneer experimental study was designed in attempt to enhance the function of external anal sphincter using injections of autologous muscle-derived stem cells. The study is designed as a prospective experimental study. It is being conducted by two cooperating research centres - the 3rd Department of General Surgery, Jagiellonian University in Cracow and the Department of Reproductive Biology and Stem Cells, Institute of Human Genetics, Polish Academy of Sciences in Poznan. We would like to present a case of the representative patient enrolled to our study.

A 20-year old male with faecal incontinence due to an old external anal sphincter rupture in a road accident was enrolled to the study. Sphincter rupture had been repaired surgically right after the accident (with an end-to-end sphincteroplasty). The patient underwent 6 months of biofeedback training after the wounds were healed. At the time of enrolment, he still complained of gas and loose stool incontinence, daily soiling, with necessity to wear pads. Endoanal ultrasound showed a $8-10-\mathrm{mm}$ scar on the left circumference of internal and external sphincter muscle, where anal canal was ruptured during the accident, and surgically repaired afterwards. Anorectal manometry showed decreased both mean resting and maximum squeeze pressure, with short high pressure zone length. Endoanal 
surface electromyography was performed using a 48channel, 3-ring endoanal probe according to routine protocol. Mean amplitude and frequency of the signal from each of 16 electrodes (corresponding to 16 cut-outs of external sphincter circumference) were assessed, in each of the three rings (a total of 48 signals in each acquisition). In the signal analysis, there was an area with no electrical activity present - the area corresponded to scar tissue - as it contained mostly fibrous tissue with potentially very few myocytes, it was unable to conduct the motoric units' action potentials (MUAPs), hence the lack of electrical activity in this area. In Faecal Incontinence Severity Index (FISI) questionnaire the patient scored 30 points.

In local anaesthesia, a $1-\mathrm{cm}^{3}$ sample of lateral head of quadriceps muscle of the thigh was harvested. There were no complications of this procedure. The tissue fragment was mechanically dissected and subjected to digestion with $0.02 \%$ collagenase solution (Sigma). The obtained cell suspension was filtered, centrifuged and plated on gelatine-covered culture dish. The myoblasts were cultured to $70 \%$ confluence and passaged every 4-5 days while the medium was replaced every other day. The myoblasts were cultured for 4.5 weeks. To confirm myogenic properties of obtained cells, their differentiation potential was also assessed. The prepared myoblasts population showed $6.44 \%$ of necrotic cells whereas we did not find any cells with apoptotic characteristics $(<0.5 \%)$. After 30 days of in vitro culture, about $6 \times 10^{8}$ myoblasts were prepared for the transplantation procedure.

After preparation of the site, the suspension of isolated myogenic stem cells was then administered into the external anal sphincter under direct visual and ultrasonographical guidance. Total volume of the suspension $\left(3 \mathrm{~cm}^{3}\right)$ was divided into three $1-\mathrm{cm}^{3}$ portions: $1 \mathrm{~cm}^{3}$ was injected on both sides of the muscle scar, one was applied on the remaining circumference of external sphincter muscle ring (several injections $1 \mathrm{~cm}$ apart), the last portion was injected directly into the scar, so that the bolus of injected fluid had direct contact with healthy muscle tissue, creating a "bridge" between muscle ends, across the scar. The procedure was performed without any complications, and the patient went home the same day.

The patient was scheduled for follow-up visits in 6-week intervals, each of which consisted of general examination, FISI questionnaire, manometry, EMG and ultrasound examinations.

Regarding the continence score, there was almost no improvement after 6 weeks, but during the following 6 weeks, patient reported gradual improvement. This correlated with increased anorectal squeeze pressures in manometry. EMG showed MUAPs gradually appearing in the scar area, where there was no visible electrical activity before implantation. Additionally, in the follow-up, overall mean and median frequency of signal was lower than before implantation - meaning that the quantity of synchronous and properly propagating MUAPs was significantly increased in the course of a total of 12 months of observation. After completion of the observation, the patient was still incontinent for flatus, but did not report any soiling nor stool incontinence, and was generally satisfied with the results of the experiment.

To sum up, presented case of the enrolled patient provided very encouraging results. The patient not only improved clinically, in terms of subjective assessment of daily stool and flatus continence-which alone might be considered a placebo effect, but also in objective functional assessment, with significant increase in anorectal manometry. The EMG analysis was particularly interesting, as it showed action potentials in regions previously lacking of conductive and contractile capabilities, which may be a "proof of concept" that implanted myoblasts not only developed into myocytes, but were able to "plug-in" to existing motoric units, creating more muscle tissue at individual motoneurons' disposal. A significant difference in mean and median frequency of electrical activity of the muscle (meaning a change in signal-to-noise ratio in favour of coordinated electrical action potentials) may also be a proof that efficiency of motoric units of the external anal muscle was significantly improved by autologous stem cells implantation.

This result must be taken as very encouraging in the light of further conducted phase I/II clinical trial.

Open Access This article is distributed under the terms of the Creative Commons Attribution License which permits any use, distribution, and reproduction in any medium, provided the original author(s) and the source are credited.

\section{References}

1. Wernig A, Schäfer R, Knauf U, Mundegar RR, Zweyer M, Högemeier $\mathrm{O}$ et al (2005) On the regenerative capacity of human skeletal muscle. Artif Organs 29(3):192-198

2. Meng J, Adkin CF, Xu S, Muntoni F, Morgan JE (2011) Contribution of human muscle-derived cells to skeletal muscle regeneration in dystrophic host mice. PLoS ONE 6(3):e17454

3. Menasché P, Hagège A, Scorsin M, Pouzet B, Desnos M, Duboc D et al (2001) Autologous skeletal myoblast transplantation for cardiac insufficiency. First clinical case. Arch Mal Coeur Vaiss 94(3):180-182

4. Simniak T, Kalawski R, Fiszer D, Klimowicz A, Kurpisz M (2002) Myoblast transplantation in the treatment of post-infarction heart failure. Kardiol Pol 57(10):354-358 